\title{
An improved algorithm for automatic detection of saccades in eye movement data and for calculating saccade parameters
}

\author{
F. BEHRENS \\ Otto-von-Guericke University Magdeburg, Magdeburg, Germany \\ M. MacKeben \\ Smith-Kettlewell Eye Research Institute, San Francisco, California \\ AND \\ W. SCHRÖDER-PREIKSCHAT \\ University of Erlangen-Nuremberg, Nuremberg, Germany
}

\begin{abstract}
This analysis of time series of eye movements is a saccade-detection algorithm that is based on an earlier algorithm. It achieves substantial improvements by using an adaptive-threshold model instead of fixed thresholds and using the eye-movement acceleration signal. This has four advantages: (1) Adaptive thresholds are calculated automatically from the preceding acceleration data for detecting the beginning of a saccade, and thresholds are modified during the saccade. (2) The monotonicity of the position signal during the saccade, together with the acceleration with respect to the thresholds, is used to reliably determine the end of the saccade. (3) This allows differentiation between saccades following the main-sequence and non-main-sequence saccades. (4) Artifacts of various kinds can be detected and eliminated. The algorithm is demonstrated by applying it to human eye movement data (obtained by EOG) recorded during driving a car. A second demonstration of the algorithm detects microsleep episodes in eye movement data.
\end{abstract}

Discrimination between saccadic and nonsaccadic components of eye movement data is a necessary first step in processing before any detailed analysis of a saccade can be performed. The signals controlling eye movements are generated in the oculomotor nuclei in the brain stem and represent a superposition of signals from different premotor centers. Velocity thresholds for saccade detection have been used in other methods (Barnes, 1982; Niemenlehto, 2009). Acceleration is well suited to distinguishing between the two functional components of eye movements, because the velocity ranges of the quickest slow eye movements and the slowest parts of saccades overlap, whereas acceleration signals show different profiles. We have demonstrated elsewhere the advantages of using the eye-acceleration signal for detecting saccades for an algorithm that can only detect and extract saccades in eye movement data (Behrens \& Weiss, 1992). That algorithm was developed specifically for analysis of eye movement data during nystagmus. It was designed to analyze repetitive eye movements of constant amplitude in a clinical test (Behrens \& Weiss, 1999); however, it could not distinguish artifacts or analyze saccade parameters, and it introduced a refractory period. To separate saccadic from nonsaccadic components of eye movements reliably, our earlier algorithm needed a fixed acceleration threshold as one input parameter. In order to automatically process a large amount of data offline, this threshold had to be selected by the operator. The absolute value of acceleration was analyzed relative to this threshold, and the end of the saccade was detected by a number of subthreshold values (equivalent to $80 \mathrm{msec}$ ). In contrast, the improved algorithm we present here is superior because it uses adaptive thresholds that are calculated from the acceleration signal itself, and it analyzes the acceleration signal in relation to the modified thresholds during the saccade. The advantage is that this reduces the number of input parameters, so that it can also be used for uncalibrated data. With the introduction of an adaptive threshold, the use of different constant thresholds depending on the paradigm becomes unnecessary. The new algorithm was implemented to detect normal saccades that follow the main sequence (Bahill, Clark, \& Stark, 1975), as well as artifacts and longlasting, slowly drifting, non-main-sequence saccades that have been called glissades (Bahill \& Stark, 1975; Weber $\&$ Daroff, 1972). These drifting eye movements with an asymmetric velocity profile occur in connection with saccades of extremely large amplitude or in sleepy subjects (Van Opstal \& Van Gisbergen, 1987). Normal saccades

F. Behrens, franklwbehrens@gmail.com 


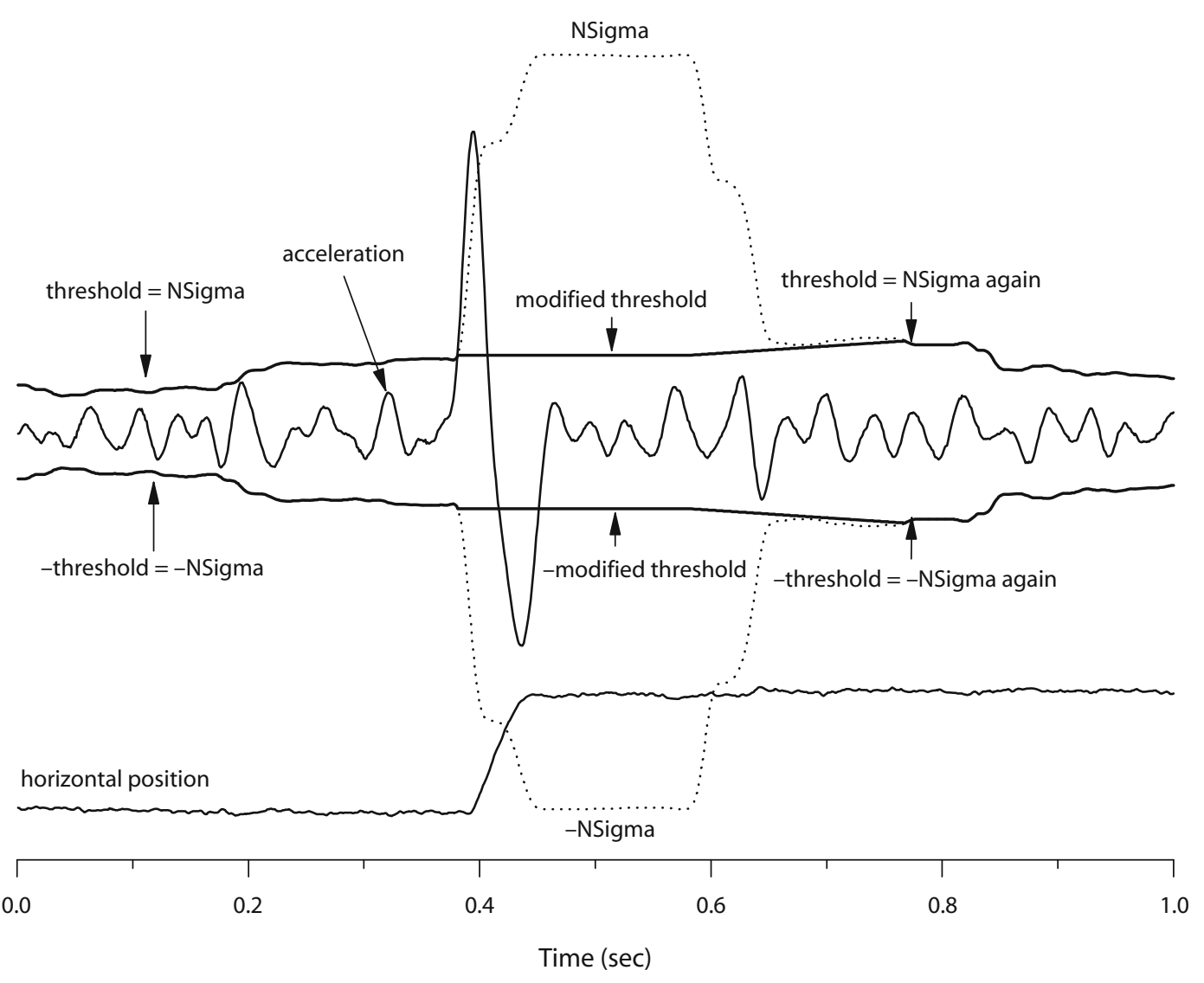

Figure 1. A horizontal leftward saccade and the calculated acceleration. The multiple (factor $N=3.4$ ) of the standard deviation of the distribution of 200 preceding acceleration values constitutes the threshold. Acceleration values of nonsaccadic eye movements are positioned between + NSigma and - NSigma. After the acceleration exceeds the threshold the first time, the threshold is modified and held constant for $200 \mathrm{msec}$. Then, the threshold increases slowly to reach a reliable point of intersection with the diminishing NSigma after a saccade. From this point of intersection on, the threshold is set to NSigma again.

are those for which the acceleration signal crosses above the positive threshold as well as below the negative threshold, or vice versa. These saccades occur most frequently, and we will refer to them here as "biphasic."

The improved algorithm presented here will be explained using horizontal eye movement data containing saccades that begin with a positive acceleration. The algorithm was developed for data obtained by electrooculography (EOG), but it has also proven useful for videobased eyetracker data. The algorithm introduced here works independent of an absolute calibration of the eyeposition signal, so that onset, duration, and direction of a saccade can be determined. Therefore, the algorithm can be used for real-time data processing (e.g., for fixation control). If the goal is to determine the absolute amplitude or maximum speed of a saccade, however, calibrated eye signals are necessary. This means that the calibration of eye-position data has to remain valid during real-time data acquisition.

\section{METHOD}

Sampling frequency was $1 \mathrm{kHz}$ for all examples shown here. The recording method was either the EOG (bandwidth DC to $100 \mathrm{~Hz}$ ) or a video-based eyetracker (Chronos Vision: OneK-plus model), whose data was compared with EOG data. The acceleration signal was calculated by convolution of the position data with a kernel $\left\{\begin{array}{lll}1 & -2 & 1\end{array}\right\}$ representing the second derivative, combined with finite impulse response (FIR) filter coefficients for low-pass filtering at $25 \mathrm{~Hz}$, and then divided by the square of the sampling interval (Behrens \& Weiss, 1992). This signal spreads the saccades as a result of the low-pass filtering, but it does not displace them with respect to their phase. During smooth-pursuit eye movements with uniform motion or during fixation, the mean acceleration is zero. However, the process generates a noisy acceleration signal in spite of the lowpass filtering by the second derivative, which is caused by the noise of the recording method (EOG data). The acceleration values follow a Gaussian distribution.

\section{Calculation of an Adaptive Threshold}

Adaptive thresholds have been used on eye movement signals before (Niemenlehto, 2009; Tole \& Young, 1981). The acceleration signal during fixation or during uniform slow-pursuit eye movements (nonsaccadic) would be zero without noise. Because of the noise, acceleration values belonging to these eye movements vary strongly and are normally distributed $(M=0, S D=$ Sigma), which we have established experimentally by analyzing fixation data. The characteristic microsaccades during fixation could not be considered here, since their amplitudes are too small to be in the range of sensitivity of the monitoring system (EOG). This is not to say that the proposed algorithm would not work on them, but it will have to 


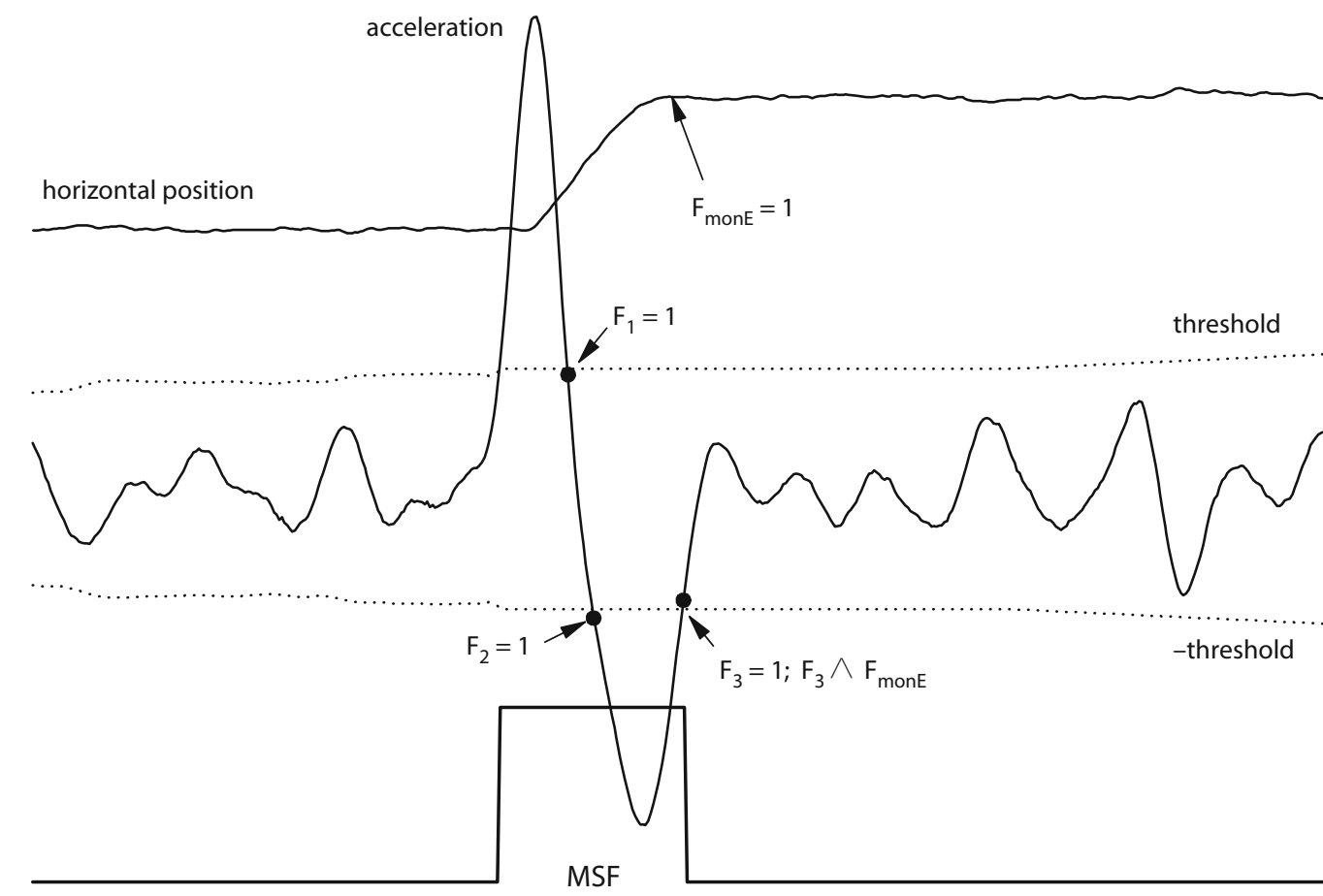

\begin{tabular}{|l|l|l|l|l|l|}
\hline & 1 & 1 & & & \\
0.0 & 0.1 & 0.2 & 0.3 & 0.4 & 0.5
\end{tabular}

Time (sec)

Figure 2. The biphasic course of acceleration during a normal saccade. Exceeding the positive threshold for the first time sets the membership function (MSF) to 1. The first decline of the acceleration below the positive threshold sets flag $F_{1}$ to 1 . The first decline below the negative threshold sets $F_{2}$ to 1 , which indicates that the signal is biphasic. The end of monotonicity of the position signal is represented by $F_{\text {monE }}=1$. After the acceleration signal exceeds the negative threshold, $F_{3}=1$. In this case, the end of monotonicity occurred earlier. To prevent a retriggering of a new saccade, the end of the saccade must be defined by the conjunctive AND of $F_{\text {monE }}=1$ and $F_{3}=1$. MSF is displayed in the bottom trace.

be tested in future research. Almost all values (more than 99.93\%) lie in the interval between +NSigma and -NSigma if $\mathrm{N}=3.4$. The empirically assessed value of $\mathrm{N}=3.4$ is a good choice for the threshold, and this value was used here throughout.

For computational reasons, the momentary threshold NSigma is determined from the Gaussian distribution of the 200 preceding acceleration data points, which is quite sufficient for this calculation. Acceleration values outside the interval +NSigma to - NSigma indicate a fundamental change (i.e., a saccade).

During a saccade, the function NSigma increases strongly (Figure 1). Because of the method of calculation of sigma, the influence of the saccade on the function NSigma does not subside before $200 \mathrm{msec}$ after the saccade has ended. Because of the increase of NSigma during the saccade, the threshold is modified during the saccade - that is, fixed at the moment the acceleration exceeds the threshold the first time. Subsequently, the slow linear increase of the threshold after $200 \mathrm{msec}$ forms a reliable intersection with the diminishing NSigma to quickly achieve the new threshold NSigma. When an artifact is detected, the threshold can be reset to NSigma instantly. For biphasic saccades, the threshold can be reset to NSigma $200 \mathrm{msec}$ after flag $\mathrm{F}_{3}$ is set to 1 . The modified threshold is the reason why, after the end of a saccade, a new saccade can be detected immediately (i.e., without a refractory time).

\section{Saccade Detection}

As in the previous algorithm (Behrens \& Weiss, 1992), we used a membership function to differentiate between saccadic and nonsaccadic components of eye movements (Arzi \& Magnin, 1989). The beginning of a saccade is defined by the sample, where the acceleration signal first exceeds the positive threshold. At this point, the membership function (MSF) is set to +1 , and a counter (countS) is started. MSF is set to -1 when the acceleration first falls below the negative threshold. If no saccade is detected, MSF $=0$. In the algorithm described here, the acceleration and position signals are analyzed during a saccade relative to the modified thresholds and the end of monotonicity. The detection of the end of a saccade is extremely important to guaranteeing proper functioning of the algorithm. Use of the acceleration or velocity signal alone for this purpose is not always successful, because the superposition with noise can interfere with finding the end of the saccade. To reliably calculate the end of a saccade, both acceleration and position signals have to be utilized. An additional advantage of this algorithm is that it differentiates between saccades of different types and artifacts, which can be rejected if necessary.

The algorithm can be explained using a main-sequence saccade, the acceleration signal of which exceeds the positive threshold for the first time, so that MSF $=+1$ (Figure 2 ). When the acceleration 

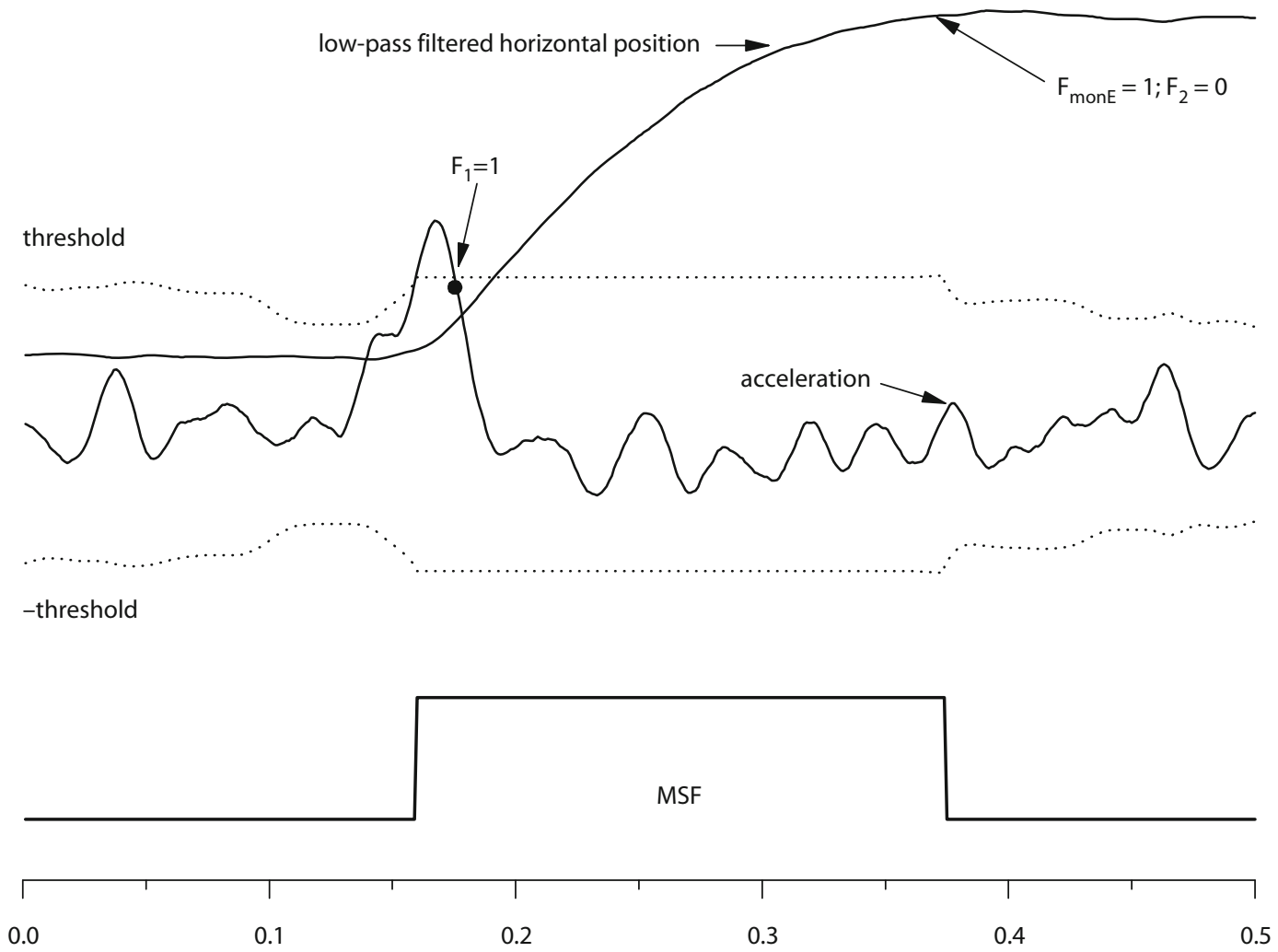

Time (sec)

Figure 3. An example of a monophasic non-main-sequence saccade of $38^{\circ}$ amplitude. The first dip of the acceleration below the positive threshold flag $F_{1}=1$ starts the monitoring of monotonicity to detect its breakdown (see the membership function [MSF] trace). Because $F_{2}=0$ and the number of sample points count $t_{S}$ is greater than 100 , the end of the non-main-sequence saccade is defined by $F_{\text {mone }}=1$. The duration is 214 msec.

signal falls below the positive threshold for the first time, a flag is set $\left(\mathrm{F}_{1}=1\right)$. This means that from this data point on, the position signal (if necessary, low-pass filtered) as a monotonically increasing function needs to be observed. At the end of monotonicity, a flag is set $\left(\mathrm{F}_{\text {monE }}=1\right)$. When the acceleration falls below the negative threshold the first time, it means that the signal is biphasic $\left(\mathrm{F}_{2}=1\right)$. The end of such a saccade is obtained both when the end of the monotonicity of the position signal is detected and when the acceleration signal is greater than the negative threshold $\left(\mathrm{F}_{3}=1\right.$; conjunctive AND ). In normal biphasic saccades, the end of monotonicity is detected before the acceleration exceeds the negative threshold. The conjunctive AND prevents retriggering, which would simulate the beginning of a new saccade. Monophasic acceleration signals are represented by $\mathrm{F}_{2}=0$. The end of a monophasic saccade is detected when the end of position monotonicity is reached $\left(\mathrm{F}_{\mathrm{monE}}=1\right)$.

Artifacts are monophasic and are characterized by a short duration (countS $<100 \mathrm{msec}$ ), whereas non-main-sequence saccades are long-lasting signals that are sometimes monophasic (Figure 3; countS $>100 \mathrm{msec}$ ). Further calculations can be performed if the end of the saccade is detected, depending on the type of saccade or artifact. If the detected signal is an artifact, the incorrect countS data $\mathrm{MSF}=1$ or $\mathrm{MSF}=-1$ have to be replaced with $\mathrm{MSF}=0$. Finally, all flags are set to zero. Because of the spreading of the acceleration signal, the duration of the saccade in the calculated MSF function is sometimes slightly overestimated.

If the goal of data analysis is real-time saccade detection, the constant delay between acceleration and position signal has to be taken into consideration. The time delay caused by the FIR filter convolu- tion is $\mathrm{M}$ times the sampling interval, whereby the number of FIR filter coefficients is $2 \mathrm{M}+1$.

\section{Calculation of Saccade Parameters}

As long as we are interested only in rejecting saccades from eye movement data, the older algorithm (Behrens \& Weiss, 1992) works sufficiently. If we were interested in saccade parameters like peak velocity, duration, amplitude, or a measure of asymmetry of the velocity profile, however, further analysis would be necessary. The analysis has to be carried out at the end of a detected saccade and before the flags are reset. The procedure is described in Figure 4 using the saccade from Figure 2 but with doubled time resolution for greater clarity.

Trace 1 in Figure 4 shows the MSF caused by the detection algorithm. In the next step, velocity profiles with different low-pass filter frequencies were calculated during the interval defined by MSF $=1$ (Figure 4, trace 2). Because the FIR filters used here produced no shifts, relative maxima of velocity $\left(\mathrm{V}_{\mathrm{rmax}}\right)$ are positioned at the same sample t0 independent of the different low-pass filter frequencies; however, the values of $\mathrm{V}_{\text {rmax }}$ depend on the low-pass filter frequencies. We used a velocity profile calculated with a filter frequency of $10 \mathrm{~Hz}$. The profile is smoothed strongly, but the $\mathrm{V}_{\text {rmax }}$ and its sample t0 can be calculated. To obtain the peak velocity of the saccade, a velocity profile that is minimally smoothed $(75-\mathrm{Hz}$ low-pass filter frequency) was used (thin line, trace 2). Only the noisy data around the sample t0 were considered for a second-order fit. Data within the interval $\mathrm{t} 1-\mathrm{t} 2$ were used for this calculation. The interval $\mathrm{t} 1-\mathrm{t} 2$ is defined by $\mathrm{V}_{\text {rmax }}$ data greater than $70 \%$. The fitting function (thick 


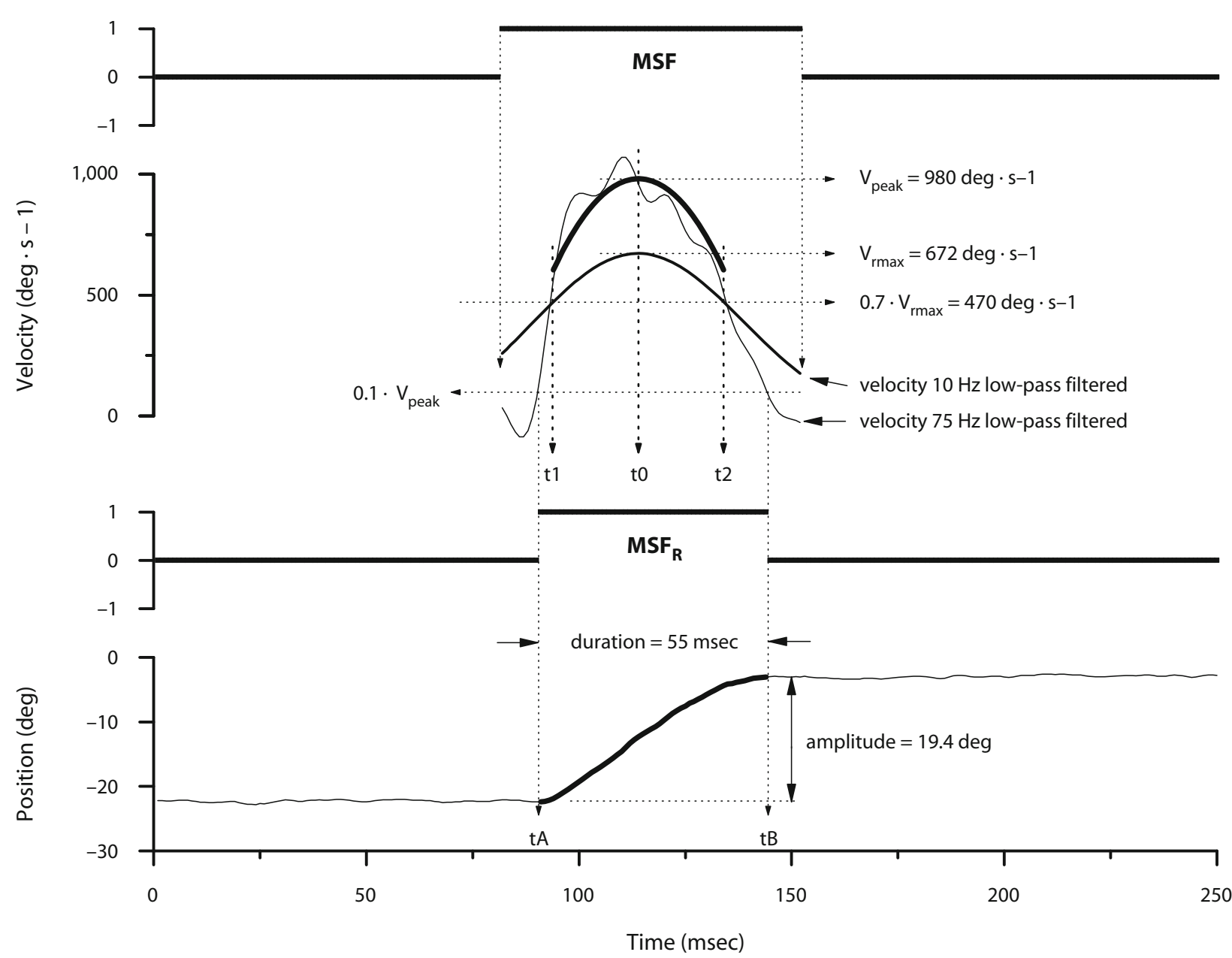

Figure 4. The parameter calculation demonstrated by the saccade from Figure $2 \mathrm{~A}$ with doubled time resolution. The membership function (MSF) from the detection algorithm is displayed in trace 1 . Only data within MSF $\neq 0$ are calculated. The 10-Hz low-pass filtered velocity is displayed to find the relative maxima of velocity $\left(V_{r m a x}\right)$ and $t 0$. The interval $t 1-t 2$ is defined by data greater than $0.7 \cdot V_{\text {rmax }}$. The 75-Hz low-pass filtered noisy velocity is fitted with a second-order function around f0 during the interval t1-t2. The absolute value of the fit represents the maximum velocity $\left(V_{\text {peak }}\right)$ of the saccade. The beginning and end of the saccade are defined by the samples $t A$ and $t B$, where the velocity becomes smaller than $0.1 \cdot V_{\text {peak }} \cdot$ Trace 3 displays the revised membership function $\left(M_{S F}\right)$, and trace 4 shows the eye position before and after the detected saccade (thin line) and during the detected saccade (thick line).

line, trace 2) symmetrically around $\mathrm{t} 0$ is $\mathrm{V}=\mathrm{A} \times(\mathrm{t}-\mathrm{t} 0)^{2}+\mathrm{C}$ during the interval $\mathrm{t} 1-\mathrm{t} 2$, where the absolute value $\mathrm{C}$ represents peak velocity $\left(\mathrm{V}_{\text {peak }}\right)$. Several definitions are used to define the beginning and end of a saccade. We defined these for biphasic saccades by the $10 \%$ values of $\mathrm{V}_{\text {peak }}$ at samples $\mathrm{tA}$ and $\mathrm{tB}$. To find these samples, we calculated starting from t0 up and down until the velocity (lowpass filtered at $75 \mathrm{~Hz}$ ) was smaller than $0.1 \times \mathrm{V}_{\text {peak }}$. However, this procedure does not work correctly for non-main-sequence saccades. The beginning of the saccade needs to be detected using the method mentioned above, and the end needs to be detected by using the sampling point at the end of the monotonicity in the position signal. After the calculation of saccade parameters, a revised membership function $\left(\mathrm{MSF}_{\mathrm{R}}\right.$; Figure 4, trace 3$)$ can be calculated between tA and $\mathrm{tB}$, which compensates for the effect of spreading caused by the use of low-pass filters that calculate the acceleration signal. In trace 4, the eye position (thin line) and the position during the detected saccade (thick line) are displayed. The parameters of the examined saccade are plotted in Figure 4. The data for amplitude and duration lay on the main sequence plots (Bahill \& Stark, 1975) but the peak velocity was higher, perhaps due to the method of calculation described above.

\section{RESULTS}

\section{Driving a Car}

The algorithm was demonstrated using horizontal eye movements recorded while a subject was driving a car during a change of direction (see Figure 5, trace 1). While the driver was turning left, the visual surround moved in the opposite direction. This strong Ganzfeld stimulus can cause a weak optokinetic nystagmus with the compensatory component to the right (Figure 5 , trace 2 ). The detected saccades are displayed in Figure 5, trace 3. MSF $=1$ detects saccades to the left, whereas MSF $=-1$ detects saccades to the right. Outside of saccades, MSF $=0$.

\section{Microsleep Episodes}

A second example demonstrating the usefulness of the algorithm is displayed in Figure 6. As described by Kuhlo and Lehmann (1964), slow, pendular, horizontal 

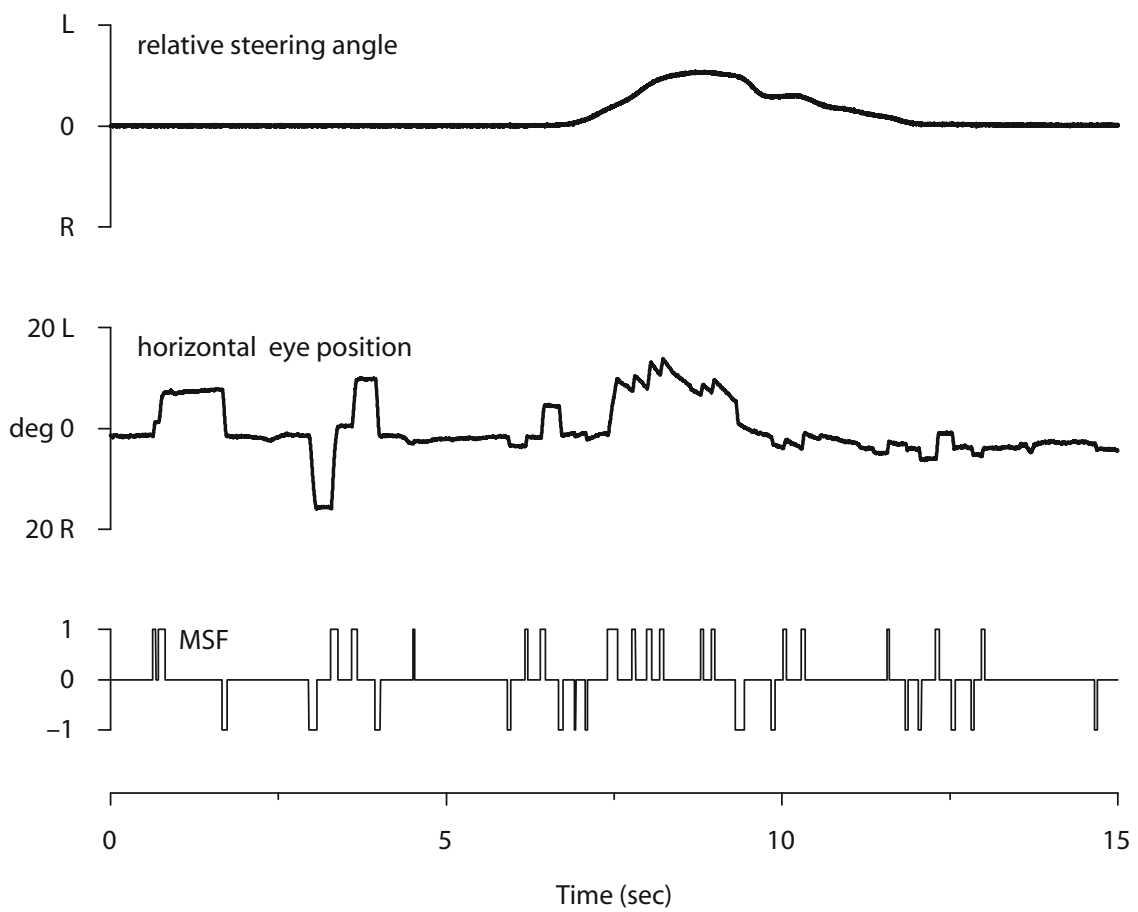

\begin{abstract}
Figure 5. Demonstration of the algorithm, using data recorded during real driving of a car. Trace 1 shows the relative steering angle while driving through a left turn. The corresponding horizontal component of eye movements with optokinetic nystagmus and compensatory movement to the right is displayed in trace 2. The calculated membership function (MSF) is plotted in trace 3.
\end{abstract}

eye movements of $20^{\circ}$ to $60^{\circ}$ amplitude and $0.2-0.6 \mathrm{~Hz}$ can be observed in most subjects during episodes of microsleep (i.e., wake-sleep-wake transitions). The described algorithm has also been used to detect such episodes. Figure 6 , trace 1 , shows the vertical eye movement component, which allows distinguishing between blinks and longer lasting lid closures as a feature of the EOG recording method. The horizontal component is displayed in trace 2. Small saccades before the microsleep episode, which is saccade-free, and postmicrosleep saccades can be discriminated. The detected saccades are plotted in trace 3 and are represented by the MSF. On the basis of this function, the postsaccadic time is displayed in trace 4 . The maxima represent the intersaccadic intervals. Because the eye position after the end of the last saccade is known, we can calculate a function that represents the sum of squares of the differences between the actual position and the position after the last saccade divided by the postsaccadic time. This function is displayed in trace 5 and can be seen as postsaccadic amplitude energy, but not in the physical sense. A signal to indicate a microsleep episode (trace 6) can be generated by empirically defining two separate thresholds for duration and postsaccadic amplitude energy, both of which have to be exceeded.

\section{DISCUSSION}

Our results have shown that the new algorithm accomplishes several points that exceed the capabilities of our older algorithm:
Automatic calculation of an adaptive threshold from preceding acceleration data

Modification of the threshold during the saccade

Determination of the end of a saccade from the end of monotonicity of the position signal during the saccade, together with the acceleration with respect to the thresholds

The adaptive threshold is independent of the kind of eye movement. Other saccade-detection methods use different constant thresholds for different kinds of eye movements (e.g., EyeLink 1000, SR Research Ltd., Ottawa, ON). Our algorithm also differentiates between saccades following the main sequence and non-main-sequence saccades, and it allows detection and differentiation of various artifacts. The twofold differentiation that is necessary to calculate acceleration from the position signal introduces additional noise and, thus, a low signal-to-noise ratio. In order to obtain a useful signal, low-pass filtering is necessary. The signal-to-noise ratio can be increased by lowering the lowpass filter frequency, but the resulting temporal spreading of the saccades in the acceleration signal can cause overlapping of consecutive saccades. Here, a low-pass filter frequency between 12 and $25 \mathrm{~Hz}$ for processing the acceleration signal was chosen as a compromise. Notch filtering of noisy signals is sometimes necessary. The small saccade indicated by an arrow in Figure 6, trace 2, has an amplitude of almost $1.5^{\circ}$, which is near the resolution threshold of the EOG method. Moreover, the new algo- 

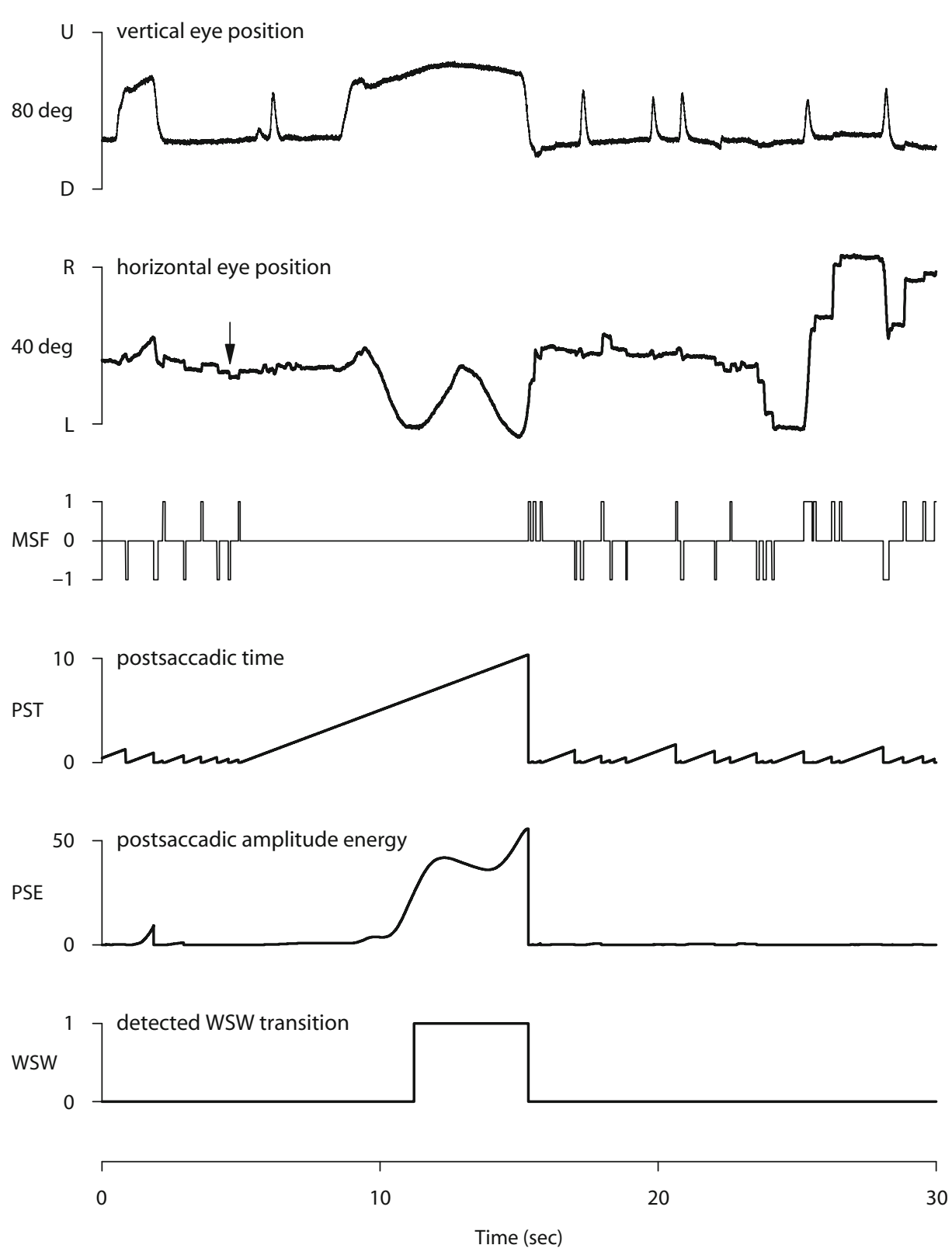

Figure 6. Demonstration of the usefulness of the algorithm by the detection of a wake-sleep-wake (WSW) transition during a microsleep episode on the basis of the analysis of horizontal eye movements (trace 2). The vertical component of eye movements is shown in trace 1 . Blinks and lid closures can be discriminated, which is a benefit of the EOG method. Small saccades of approximately $1.5^{\circ}$ (arrow in trace 2) are detected in the horizontal component. The membership function MSF is plotted in trace 3, which is the basis for the calculation of the postsaccadic time (PST; trace 4). Postsaccadic amplitude energy (PSE), the squared sum of differences of the actual amplitude and the amplitude at the end of the last saccade divided by the postsaccadic time, is shown in trace 5. The detected microsleep episode is indicated in the bottom trace.

rithm needs no input of parameters to calculate an adaptive threshold and can use even unscaled data for saccade detection.

Furthermore, the algorithm can detect and suppress various small artifacts. It can discriminate between two kinds of artifacts: (1) Artifacts that occur during the discrimination algorithm caused by short, small monophasic acceleration signals crossing a threshold. (2) Artifacts that can be detected during the following analysis of a saccade. Small saccades near the resolution threshold of the method of registration $\left(1^{\circ}\right.$ to $2^{\circ}$ in EOG) often show irregular velocity profiles and can be detected and eliminated if necessary.

The connection between sleep, eye movements, and traffic was recognized very early (Miles, 1929). Since then, there have been numerous publications concerning 
microsleep and driving hazards (e.g., Connor, Whitlock, Norton, \& Jackson, 2001; Lyznicki, Doege, Davis, \& Williams, 1998; McCartt, Rohrbaugh, Hammer, \& Fuller, 2000).

Our new algorithm also works on data from other eyetracking methods. Blinks or long-lasting lid closures cause data loss in video-based eyetracker data. In order to obtain a continuous time series, the undefined interruptions in the data stream need to be replenished by interpolation.

In summary, the improved algorithm introduced here is a tool for the automatic detection and analysis of saccadic eye movements. It makes the identification of saccades of different types and the differentiation of artifacts more accurate and more reliable.

\section{AUTHOR NOTE}

Correspondence concerning this article should be addressed to F. Behrens, Otto-van-Guericke University Magdeburg, Institute for Distributed Systems, Department of Embedded Systems and Operating Systems, Universitätsplatz 2, D-39106 Magdeburg, Germany (e-mail: franklwbehrens@gmail.com).

\section{REFERENCES}

Arzi, M., \& Magnin, M. (1989). A fuzzy set theoretical approach to automatic analysis of nystagmic eye movements. IEEE Transactions on Biomedical Engineering, 36, 954-963.

Bahill, A. T., Clark, M. R., \& Stark, L. (1975). The main sequence: A tool for studying human eye movements. Mathematical Biosciences, 24, 191-204.

BAHILL, A. T., \& STARK, L. (1975). Overlapping saccades and glissades are produced by fatigue in the saccadic eye movement system. Experimental Neurology, 48, 95-106.

BARNES, G. R. (1982). A procedure for the analysis of nystagmus and other eye movements. Aviation, Space, \& Environmental Medicine, $\mathbf{5 3}, 676-682$.
Behrens, F., \& Weiss, L.-R. (1992). An algorithm separating saccadic from nonsaccadic eye movements automatically by use of the acceleration signal. Vision Research, 32, 889-893.

Behrens, F., \& Weiss, L.-R. (1999). An automated and modified technique for testing the retinal function (Arden test) by use of the electrooculogram (EOG) for clinical and research use. Documenta Ophthalmologica, 96, 283-292.

Connor, J., Whitlock, G., Norton, R., \& Jackson, R. (2001). The role of driver sleepiness in car crashes: A systematic review of epidemiological studies. Accident Analysis \& Prevention, 33, 31-41.

Kuhlo, W., \& Lehmann, D. (1964). Das Einschlaferleben und seine neurophysiologischen Korrelate. Archiv für Psychiatrie und Zeitschrift für die gesamte Neurologie, 205, 687-716.

LyzNicki, J. M., Doege, T. C., Davis, R. M., \& Williams, M. A. (1998). Sleepiness, driving, and motor vehicle crashes. Journal of the American Medical Association, 279, 1908-1913.

McCartt, A. T., Rohrbaugh, J. W., Hammer, M. C., \& Fuller, S. Z. (2000). Factors associated with falling asleep at the wheel among long-distance truck drivers. Accident Analysis \& Prevention, 32, 493504.

MiLES, W. R. (1929). Horizontal eye movements at the onset of sleep. Psychological Review, 36, 122-141.

Niemenlehto, P.-H. (2009). Constant false alarm rate detection of saccadic eye movements in electro-oculography. Computer Methods \& Programs in Biomedicine, 96, 158-171.

Tole, J. R., \& Young, L. R. (1981). Digital filters for saccade and fixation detection. In D. F. Fisher, R. A. Monty, \& J. W. Senders (Eds.), Eye movements: Cognition and visual perception (pp. 247-256). Hillsdale, NJ: Erlbaum.

Van Opstal, A. J., \& Van Gisbergen, J. A. M. (1987). Skewness of saccadic velocity profiles: A unifying parameter for normal and slow saccades. Vision Research, 27, 731-745.

Weber, R. B., \& Daroff, R. B. (1972). Corrective movements following refixation saccades: Type and control system analysis. Vision Research, 12, 467-475.

(Manuscript received August 23, 2009; revision accepted for publication March 22, 2010.) 